\title{
Water Quality Model Integration in a Decision Support System
}

\author{
L. León, N. Kouwen, G.J. Farquhar, E.D. Soulis \\ Engineering, University of Waterloo, Waterloo, Ontario, Canada \\ email:Ifleonvi@uwaterloo.ca
}

Key words: Spatial decision support system, nonpoint source pollution, model integration

Abstract: $\quad$ This paper describes an integral system to model nonpoint source pollution in surface waters. Diffuse pollution models were included in a Spatial Decision Support System (SDSS) with a unique platform, common interfaces and Geographic Information System (GIS) capabilities. This consists of pre- and post-processing tools, model control and sensitivity analysis for the parameters in the models. Particularly, the construction of the interface for WATFLOOD (flood forecast hydrological model) and its link with the decision support system RAISON (Regional Analysis by Intelligent Systems On microcomputers) is presented. A water quality component was coupled to the model in order to deal with sediment and nutrient transport. Previous work with the AGNPS (Agricultural Non-Point Source) model is used for comparison with the results from the water quality component coupled to the WATFLOOD model.

\section{INTRODUCTION}

Nonpoint source pollution (NPS) models were included in a Spatial Decision Support System (SDSS) for a unique platform, common interfaces and Geographic Information System (GIS) capabilities. An integral approach is developed involving the linkage of the AGNPS (Agricultural Non-Point Source Model) and WATFLOOD (flood forecast hydrology model) with RAISON (Regional Analysis by Intelligent Systems On microcomputers) to form a SDSS to deal with NPS modeling. Further work was done to couple a water quality component into WATFLOOD that will allow calculations of sediment and nutrient transport associated to the hydrology of the model.

The original version of this chapter was revised: The copyright line was incorrect. This has been corrected. The Erratum to this chapter is available at DOI: 10.1007/978-0-387-35503-0_29 
Particularly, the construction of the interface for WATFLOOD and its link with RAISON is presented.

\section{MODEL DESCRIPTION}

WATFLOOD has been developed by Prof. N. Kouwen at the University of Waterloo. It is an integrated set of programs to forecast flood flows for watersheds having response times ranging from one hour to several weeks. The emphasis of the WATFLOOD system is on making optimal use of remotely sensed data. Radar rainfall data and landcover data from remote sensed imagery can be directly incorporated in the hydrologic modeling.

WATFLOOD is unique in its ability to preserve the distributed nature of a watershed's hydrologic and meteorological variability without sacrificing computational efficiency. This has been accomplished through the use of Grouped Response Units (Kouwen et al., 1993), in which process parameters are tied to landcover thus facilitating the transfer of model parameters from basin to basin. WATFLOOD has been designed since its inception to take advantage of weather radar and satellite imagery. The high spatial and temporal resolution of rainfall is taken into account when using radar data to estimate precipitation.

The fundamentals and equations used in the model to convert the radar reflectivity values into rainfall rates can be found elsewhere (Kouwen, 1988). In general terms, these consist of different processes to adjust the radar data for clutter, beam-blocking and attenuation, and can compute rainfall rates with values of radar reflectivities using an exponential relationship.

\section{THE WATER QUALITY COMPONENT FOR WATFLOOD}

Further work was done to couple a water quality component to WATFLOOD to allow calculations of sediment and nutrient transport associated with the hydrology of the model. A simple sediment yield model for single storm events (Hartley, 1987), was successfully used. It calculates the sediment transport capacity and the potential sediment supply, choosing whichever is less, and routing such value downstream. The sediment transport capacity is calculated using shear stress relationships.

The methods selected to simulate the nutrient processes in the water quality component for the WATFLOOD model are the same as the ones used in AGNPS. The algorithms were developed by Frere et al. (1980) and adapted to the landcover approach of WATFLOOD. Further details can be found in the technical documentation on nutrients from the AGNPS model 
(Young et al., 1986). The routing of sediments and nutrients was carried out using a mixing cell model based on the continuity equation.

The mixing cell approach is used here as a first approximation for the transport module in order to create a complete system. If future research is devoted to the water quality component, more elaborate transport models can be easily incorporated into the routing process. Deposition for sediments and the decay in the case of nutrients are estimated as a fraction of the transported mass.

\section{RAISON AS THE SDSS PLATFORM}

RAISON is a software package developed at Environment Canada's National Water Research Institute (Lam et al., 1994). It is a geographical data analysis toolkit which integrates database, spreadsheet and graphic interpretative tools with GIS and Expert Systems capabilities for microcomputers. The system provides an intuitive environment for displaying data and analysis results in the context of local geography. Though RAISON is not a full GIS nor a stand alone database, it provides enough functionality to link them. It accepts files from most commercial GIS and database systems and allows the customization of the software for any specific application.

One of the features of RAISON important to this work, is its capability to incorporate modeling tools into the system. This is done by building interfaces that interact with existing models and that can intercept the input and output to connect them to the database in the system. The communication with RAISON is done through dynamic data exchange (DDE) methods. Most of the functions used in the interface are available in dynamic-link libraries (DLL), which are libraries of routines loaded and linked into applications at run time. These functions, written specifically for RAISON, include routines for standard application tasks such as creating database files, creating grid layers, retrieving and changing database values, drawing maps and handling user actions.

\section{DATA REQUIREMENTS (DEM AND MAP FILES)}

The major asset of the GIS approach is the automatic extraction of cell related data to calculate the variables that depend on topography, soil type and landuse information. RAISON works with relational databases. The different layers on these databases represent different sources of digital information such as Digital Elevation Model Data (DEM), soil and landuse. In general terms, three sources of information are required to take full advantage of the automatic extraction of input data: DEM, soil and landuse 
data. The DEM data consist of elevation data for each element, flow direction and flow accumulation. Normally the basic information of a DEM includes the elevation of each element for the full extent of the study area.Some commercial applications include a conditioning process to create depressionless data and to calculate flow directions and flow accumulation values.

The map data includes the soil type and landcover information. Both data sources consist of a number of polygons with attached attributes. In the case of soil type the attribute of interest, in order to perform the data extraction, is the soil texture. For the landuse layer, the field to use in the extraction is the landcover attribute of the polygon.

\section{THE WATFLOOD INTERFACE}

Communication links between the interfaces and RAISON were established by using the DDEs methods and DLLs libraries for the layer functions. The interaction between the interfaces and the models is done through interchange of data via ASCII files and calls to executable files. The pre-processing tools provide easy data compiling for the models. Using the topography, soil type and land use maps in vector formats, procedures were developed to automate as much data input as possible. A control panel for model operation helps in the setup and simulation by triggering the model to run by creating a shell that activates the model. Post-processing for output data by means of graphical tools assists with the interpretation of model results. Figure 1 shows a schematic representation of the linkage between the models and RAISON through the use of the interface.



Figure 1. Schematic representation of the linkage between the models and RAISON 
All the data required for the model are stored in a database file that can contain several grids. So the first step is to prepare a file and create the grids for the model. This is done with the grid generator (Make/Edit Grid, Initialize Database and Create/Edit Grid) that allow a basic grid to be created and saved in an existing database file.

After the grid is generated and the required database structure is created in order to hold the data for the model, the next step is to start capturing the input data. The initial watershed data are captured directly into an input form. The cell data are automatically extracted from digital maps. The procedures are designed to perform the calculations and extract DEM and Map data directly into the model. Once the data have been captured and/or automatically collected from the maps and stored in the database file, the next step is to run the model and visualize the results. In order to run the WATFLOOD model, the data stored in the database have to be exported to an ASCII file in a format that the model can understand. This export and the running of the model is done through the Run toolbar. In this toolbar there are three procedures that write the ASCII file, access the windows version of the model and activate the WATFLOOD utilities. Figure 2 is an example of the interface showing the main control window with the flow editor and the directions results from the DEM extraction process.

\section{APPLICATION IN DUFFINS CREEK}

This application focuses on Duffin's Creek, a $293 \mathrm{~km} 2$ watershed draining into Lake Ontario at Ajax, $10 \mathrm{~km}$ east of Metropolitan Toronto. The landuse in the basin is mainly non-intensive agricultural with growing urban areas in the lower parts of the watershed at Pickering and Ajax. Towards the western area is Stouffville, with a flood control reservoir and a water treatment plant that discharges into the Duffins Creek west river system. Eight events from April to November 1995 were selected to test the models. 


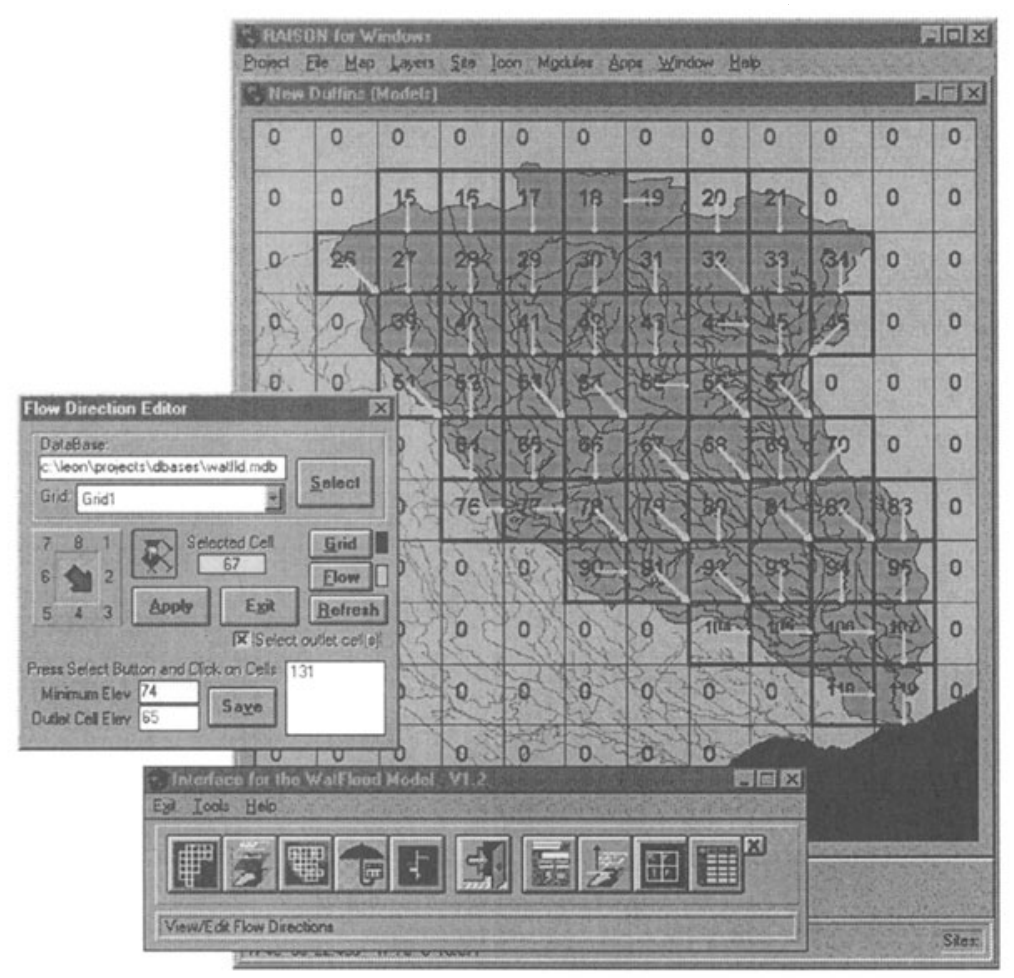

Figure 2. WATFLOOD Interface with flow directions from the DEM extraction

Two different grid sizes were created for each model (AGNPS and WATFLOOD). The first grid for the AGNPS model is a $1 \mathrm{~km}$ cell size with 205 cells and the second grid is a $2 \mathrm{~km}$ cell size with 57 cells. For the WATFLOOD model, two grids were one with a $2 \times 2 \mathrm{~km}$ cell size and a second one with a $4 \times 4 \mathrm{~km}$ size. The digital map files used for the data extraction were the same for both models. The models were run and the output was processed for the two gauging stations in the watershed. Flow results from WATFLOOD simulations allow comparison of hydrographs for the whole event. Figure 3 shows the excellent comparison between measured and calculated flows for the event of November 8-12, 1995.

Figure 4 compares predicted peak flows from the $2 \times 2 \mathrm{~km}$ AGNPS and WATFLOOD grids with the measured peak flows. In the same figure, the sediment yield using both models is also presented. In this case the comparison is between model output due to the lack of measured sediment values for the tested events. As can be seen from the simulations, calculated peak flows match extremely well the measured values for peak flow using both models. For the calculated sediment yields comparison the results 
between models are almost the same for any combination of grid sizes and they match considerably well for the tested events.
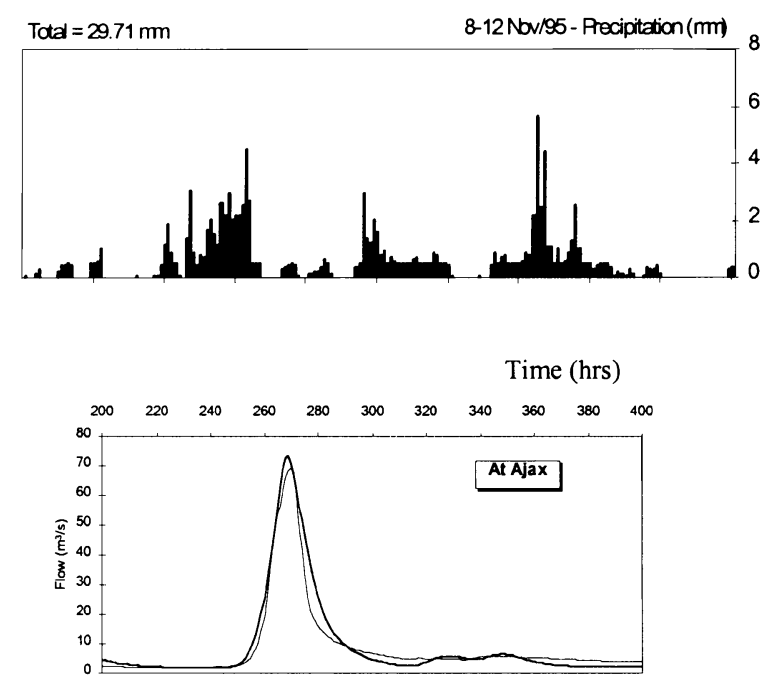

Figure 3. WATFLOOD results for November 8-12, 1995

\section{CONCLUSIONS}

The developed interfaces provide input data based on digital information of the watershed and facilitates the task of setting up a region to be modeled. The results of the simulations support the fact that, if the input parameters are well established, the models will perform close to reality in many situations. Good matches between observed and calculated values for peak flows were obtained. The fact that the models calculated sediment yields compare well is encouraging and can led eventually to an improvement in the modeling of non-point source pollution. It is worth mentioning that no major calibration was performed. 


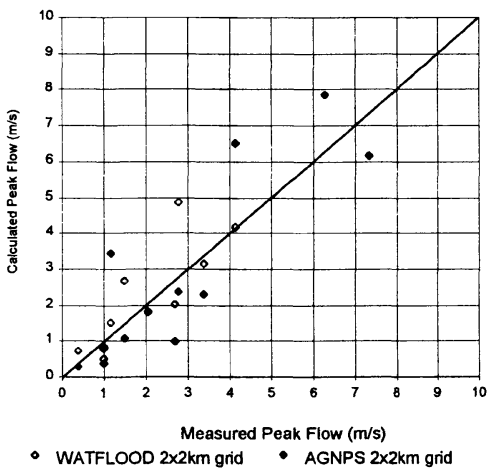

Figure 4. WATFLOOD and AGNPS results comparison for peak flows and sediment yields

\section{ACKNOWLEDGMENTS}

CONACyT (Mexican Council for Science and Technology) has sponsored the scholarship of the author. Interactions with RAISON were developed by J.D. McNeil and J. Berardine. Thanks to Gary Bowen and others at the Ontario Ministry of the Environment and Energy for supplying data, support and advice.

\section{REFERENCES}

Frere, M.H., Ross, J.D., and Lane, L.J. (1980). The Nutrient Submodel. In: Knisel, W.G. (ed.) 1980. CREAMS: A Field Scale Model for Chemicals, Runoff, and Erosion from Agricultural Management Systems, US Dept. of Agriculture, Cons. Research Report No. 26, pp. $65-86$

Hartley, D.M., (1987). Simplified Process Model for Water and Sediment Yeld from Single Storms, Transactions of the ASAE, Vol. 30, No. 3, pp. 718-723.

Kouwen, N., (1988). WATFLOOD: A Micro-Computer Based Flood Forecasting System Based on Real-Time Weather Radar, Canadian Water Res. Jr., Vol. 13(1), pp. 62-77.

Kouwen, N., Soulis, E., Pietroniro, A., Donald, J., and Harrington, R., (1993). Grouped Response Units for Distributed Hydrologic Modeling, Jr. Water Resources, V.119, No. 3, pp. 289-305.

Lam, D.C., Mayfield, C., Swayne, D.A. and Hopkins, K., (1994) A Protytype Information System for Watershed Management and Planning, Jr. Biological Sys., Vol. 2, No. 4, pp. 499-517.

León V., L.F., Lam, D.C.L., Swayne, D., Farquhar, G., Soulis, R. (1997) "Integration of a Nonpoint Source Pollution Model with a Decision Support System", 2nd. International Symposium on Environmental Software Systems, ISESS97, Whistler, BC, Canada, 1997.

Young R., Onstad, C., Bosch, D., Anderson, W., (1986). Agricultural Nonpoint Source Pollution Model: A Watershed Analysis Tool, Model Documentation, Agricultural Research Service, U.S. Department of Agriculture, Morris, MN. 